Chirurg 2011 · 82:80

DOI 10.1007/s00104-010-1995-2

Online publiziert: 24. Oktober 2010

(c) Springer-Verlag 2010

\title{
Sind Ethikfachleute im chirurgischen Alltag notwendig?
}

\section{Zum Beitrag}

Mols AM, Reiter-Theil S, Oertli D, Viehl CT

(2010) Futility: ein Begriff im chirurgischen

Alltag? Chirurg 81:643-646

\section{Leserbrief}

\section{E. Gemsenjäger \\ Basel}

Dieser Beitrag ruft zu einem Kommentar „Chirurgie quo vadis“ auf, denn er zerredet Selbstverständliches und Grundlegendes.

Die Grundlage einer optimalen Versorgung des Patienten ist in erster Linie die chirurgisch ärztliche Kompetenz. Durch den primären Beizug nichtärztlicher (Ethik-)Spezialisten würde sie eingeschränkt und verdrängt.

Konkret: Bei der betagten und dementen Patientin mit chronisch subakutem und zunehmendem Dünndarmileus ist vom erfahrenen Arzt eine peritoneale Karzinose in die Differenzialdiagnose einzubeziehen und damit eine schwierige und fragliche chirurgische Therapierbarkeit in Rechnung zu stellen. Allein der verantwortliche Chirurg (Arzt) muss diesen diagnostischen Aspekt im Kontext mit zahlreichen weiteren Faktoren gewichten, vielleicht unter Beizug eines weiteren klinisch diszipliniert denkenden, erfahrenen und menschlich (d. h. auch ethisch) kompetenten und verantwortlichen Kollegen. Bei den Entscheiden helfen kann da schon gar nicht ein medizinischer Laie. Ist denn die mitentscheidende ethische Fachperson in der Lage, zu erwägen, was der Entschluss 1. zu einer Operation und 2. zur jejunokolischen Anastomose bedeu- tet (chirurgische Morbidität, Dünndarmstuhl, Inkontinenz, Ernährungsdefizit, sollte alles denn funktionieren und sollten sich die vielleicht in sich geschlossenen Schlingen tatsächlich entleeren)? Das Abwägen ist auch für den Fachmann schwierig; "qualvoller Tod im Ileus" tönt jedoch unerlaubt laienhaft.

Was bringen da die geforderte ethische Fallbesprechung und „Aufarbeitung“ im nach Leitlinien strukturierten Gespräch, die Beschäftigung mit „Futility“, Studien über das Risiko von Burnout für den mit Alltagsforderungen konfrontierten Chirurgen? Solche Diskussionen können sich leicht am Essenziellen vorbei in Selbstzweck erschöpfen.

Qualifizierte Entscheide müssen auf ärztlichem Wissen und Erfahrung beruhen. Sie lassen sich in disziplinierter Analyse offenlegen („aufarbeiten“), beruhen aber auch auf intuitiver Gewichtung: Nutzung jenes reichen, neuronal gespeicherten und vernetzten, abrufbaren Erfahrungs- und Fachschatzes.

Der Arztberuf hat inhärent eine ethische Dimension und diese beansprucht in der Ausbildung eine angemessene Berücksichtigung. Teilung und Abschieben von Verantwortung bei alltäglichen Entscheiden durch Beizug außenstehender, theoretischer Meinungen stellt eine optimale (auch angemessen unkomplizierte und kostenverantwortliche!) Betreuung von Patienten in Frage.

\section{Korrespondenzadresse \\ Prof. em. Dr. E. Gemsenjäger \\ Gellertstrasse18, \\ CH-4052 Basel, Schweiz \\ gemsen@bluewin.ch}

